\title{
Design and Analysis of UHF RFID Tag using MATLAB
}

\author{
Aaron Don M. Africa, Phillip Christian C. Cardino, John Rossi Rafael R. Llaga \\ Department of Electronics and Communications Engineering \\ De La Salle University, Manila \\ 2401 Taft Ave., Malate, Manila 1004, \\ Philippines, aaron.africa@dlsu.edu.ph
}

\begin{abstract}
RFID is one of the biggest electronics markets being used in several industries and is responsible for many communication networks and systems. This project proposed to create a program using MATLAB to simulate a function which is like a RFID tag and scanner. The generated program was made with the help of the antenna toolbox from MATLAB. With this toolbox custom antennas were which means it was possible to generate unique antenna geometry and meshes, along with being able to determine the required impedance. Antenna Toolbox and PDE Modeler is a feature MATLAB provides where it includes commands to see the parameters in designing and analyzing RFID networks.
\end{abstract}

Key words: RFID Tag, MATLAB, RFID designs, Antenna Toolbox, PDE Modeler.

\section{INTRODUCTION}

RFID, or Radio Frequency Identification refers to a technology by which the reader captures digital data encoded in RFID tags or smart labels through radio waves [1]. RFID is identical to barcoding, in that data is retrieved from either a tag or mark by a computer that stores data in a database. Yet they have many advantages over systems using barcode asset tracking software.

RFID systems rely on radio signals to deliver information and receive it between a tag and a reader. A RFID reader sends signals to an RFID tag at its simplest level and the tag returns an information-carrying signal. They can also be designed to work on different ranges, working from low frequency, high frequency, and ultra-high frequency [2].

In this paper, the group will be simulating a UHF RFID system. So how does a UHF RFID system work? The RFID reader emits specified frequency radio waves through RFID antennas. The waves give power to the tags so that they can interact by issuing a unique identifier. They do not need batteriesand can be used for years to come. The reader analyses and gives sense to the data so that it is possible to incorporate them into an application. The standard read range is between 0 and 12 meters. Gen2 UHF RFID system composed of tags as readers, antennas, and printers.
The following are the main components of a UHF RFID system: Antennas, Labels, and Readers.

Antennas are responsible for emission and obtaining waves that enable RFID tags to be detected. When the antenna field is crossed by an RFID chip it is initiated and a signal is emitted. The antennas create various wave fields and cover various distances. The readers are the ones responsible for detecting if the RFID chip is within range, and thus activating the function it is programmed to do. As for the tags, as they come in close contact with the reader, energy is emitted from it, thus making the connection to the reader.

\section{STATEMENT OF THE PROBLEM}

RFIDs can be used in different ways. It can vary in size, shape, or design depending on what it will be used for. Location, size, orientation, and reading angles are some of the factors to be considered when designing a RFID tag [3]. The general problem when attempting to create a UHF RFID system is figuring out the right measurements of the components and deciding on the proper material to use.

With the measurements and material being considered, another problem that can arise within the subject would be budgeting. As for the requirement of multiple systems, it would be wiser to design in a smaller size of tags [4]. Since creating algorithms for the program function and antennas can be easy, the decisions that consider the qualities of the hardware will be the main focus if the need for it has arisen.

\section{BACKGROUND OF THE STUDIES}

The RFID was first established when the radar was invented in the United States. It was founded along with radio and broadcast technology. Just like every other invention, more people continue to find unique ways in improving the use of such devices. These devices were used in the war, different countries have improved them in their own way. At first, they were mainly used for communication, and soon they were used to detect enemy aircraft, and later found out to be able to detonate explosives. 
RFID systems have been used in vehicles, in such a way that they would be used as a means for payment, or identification. [5]. This technology is often used in expressways in the Philippines, or in private parking lots. These developments lead to a brighter idea in the making of smart cities.

These systems are also used in stores, supermarkets, etc. They are used as an anti-theft system that sets the alarm off when a tag from an item that has not been paid for is brought within a set range. It is not just for anti-theft, but for keeping count of inventory as well. The system works as each unique tag for a certain item is scanned and the device counts and stores the data if ever new stock has come or has been bought.

Since the RFID is usually used for identification, schools, offices, factories, etc. use it to properly distinguish the authorized people. For example, in offices and laboratories, certain areas can only be accessed by people that have a certain ID card, which contains a unique RFID tag which only the chosen people have. The more basic example is to open the doors to go to the main building using one's ID.

\section{SIGNIFICANCE OF THE STUDIES}

The findings of this study will redound to benefit security measures in the future. The use of RFID is widely known to benefit more in terms of security, than to be used as weapons that can help in war and violence. While security decreases the chance of harm, it can also be said the RFIDs can increase the level of defense within a certain structure or system. Companies and schools that use the findings to figure out unique ways to use the system can help in creating even more theories to further improve it. These improvements could be in ways to increase security within their premises. As for public transportation, like the train tickets that are commonly used, it is possible for them to create a more responsive and portable means for passengers for their convenience. Supermarkets and convenience stores, Telcos, etc. can also use this technology. Payments can be made easier using a temporary card which can act similarly to a credit card. The only difference is that you must put credits into it first and it can only be used in certain stores depending on how it is programmed. To future research on this topic, the findings may help in developing more theories that can help communities better than the ones that have been mentioned above.

\section{DESCRIPTION OF THE SYSTEM}

The planar tag is commonly a dipole tag with T-matched impedance. T-Section is one of the favorable impedance matching and is the dynamic influence in design the RFID Tag. The microchip for the tag may be a commercial tag chip that is affected by the target frequency. In MATLAB, the feed microchip has its own properties such as material and impedance and is configurable, if desired. Figure 1 shows the RFID Tag equivalence.

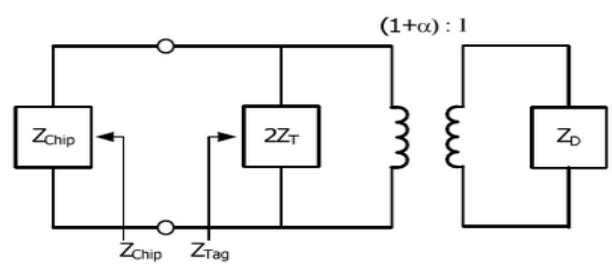

Figure 1:RFID Tag T-Matched Equivalent

\section{METHODOLOGY}

The process was to create a design of functional RFID Tag, to create a system that is matched to the designed antenna and to analyze its parameters. MATLAB was used to design and analyze the RFID circuit. This programming platform can generate meshes, necessarily the RFID Tag, using the PDE Modeler from the PDE Toolbox. Figure 2 shows the flow chart of the methodology.

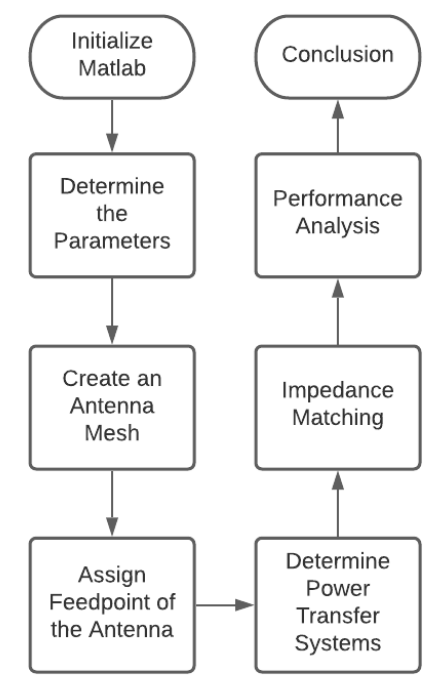

Figure 2:Methodology Flowchart

\subsection{Determining the Parameters}

The RFID Antenna should be able to provide power to the chip when receiving a certain range of frequencies.

\subsection{Generating Mesh}

In making the mesh, PDE Toolbox was used. This feature allows to draw and alter the shape of the tag to be used in the RFID network.

\subsection{Importing the mesh and Adding Feed}

The PDE Modeler allows importing the mesh to the main MATLAB module. It may be turned into an antenna by adding a feed. This can be done manually, since it is in the capabilities of the program. In this case, the command custom Antenna Mesh is used. This converted the mesh into an antenna with a characteristic preset that details its compoundings and materials, as well as providing a feedpoint to the design. 


\subsection{Power Transfer and Impedance Tuning}

With the feature provided by the Antenna Toolbox, MATLAB is able to calculate the impedance of the custom antenna. To get the best power transfer, the input impedance must match the characteristic impedance of the circuit. The programming platform is capable of adjusting the impedance properties of the antenna circuit. To determine the power delivered to the load, the command sparameter was used. This may be used to show the behavior of the power transfer given the frequency model. Impedance tuning is done after getting the reactance of the antenna. The objective is to eliminate the reactance at the target frequency of the chip. The tuning circuit is the basis for the transponder.

\subsection{Antenna Analysis}

The parameters to identify were the antenna gain, radiation pattern and Voltage Standing Wave Ratio (VSWR). MATLAB has all these functionalities present in its provided toolbox. For antenna gain and radiation pattern, the results were determined using the pattern code. The former may be also obtained from sparameter. VSWR was acquired using vswr.

\section{REVIEW OF RELATED LITERATURE}

Babaeian, F. and N. C. Karmakar [6] proposed to build cross-polar resonators and unit cells showing a clear reflection irrespective of the tag orientations. The simulation and measurement results of a few tests of the tags proposed are illustrated to illustrate the feasibility of using the tags proposed in a device with reflective objects in the background.

Polvara R., et al [7] Suggested a framework online coverage algorithm called Next-Best-Sense (NBS), an extension of the Exploration Algorithms Next-Best-View class which optimises the exploration task by balancing multiple criteria. The issue of finding all Radio Frequency Identification tags with a mobile robot is solved with NBS.

Hatem, E. et al. [8] presented an alternative analysis based on the Multiple Input Single Output (MISO) protocol to increase the precision of low-cost RFID localisation. Although most conventional systems use a single tag for translation, the architecture proposed promotes the use of a group of RFID tags called as a constellation.

Pawlowicz, B. et al. [9] discussed the application of Radio Frequency IDentification ( RFID ) technology when it comes to vehicle recognition in traffic management systems. Different Automated Vehicle Identification (AVI) system configurations have been proposed including implementations that can be used in urban traffic as part of the Smart City concept. To characterize the actions of multiple moving vehicle identifications a dynamic identification operating model is defined using RFID.
Zahran, E. et al. [10] proposed a Mixed BBO Migration (MBBOM) self-learning (SL) strategy to adjust the HIW-BBO algorithm inside an algorithm named Self Learned Invasive Weed-Mixed Biogeography Based Optimization (SLIWMBBO). The implementation of the developed SLIWMBBO model is analyzed with both the HIW-BBO and the Self Adaptive Cuckoo Search optimization algorithms by a set of 13 benchmark functions. The results of this analysis support the use of the SLIWMBBO as an enhancement algorithm to solve the complex problems.

Das, T. K. et al. [11] introduced a single feed, miniaturized, circularly polarized microstrip patch antenna that uses Koch fractal geometry for RFID applications. The framework consists of a notched square-shaped patch with the fractal edge and two sets of grounded metal strips which are capacitively coupled.

Hailemariam, Z. [12] presented a paper that proposed a novel knowledge-based Query Tree with Shortcut and Couple Resolution (QTSC) protocol to reduce the recognition delay with such a database in RFID systems. A knowledge-based query tree was first built in the proposed scheme to retain the necessary queries to classify all possible tags in the database. Then, the tags actually appear among possible tags when defining existing tags, bypass and pair-resolution techniques are employed to skip repetitive queries in the query tree and send two ID prefixes simultaneously within the same space, respectively.

\section{THEORETICAL CONSIDERATIONS}

RFID visual feature greatly influences the effectiveness of the antenna. It bases on several parameters that are required to be established. In practice, many commercial tags make use of a wide strip line as antenna body and to achieve broad bandwidth, a sizable loading bar is used. For the mesh creation, dividing the dimension is an effective way to use in designing an effective antenna. Parameters to be defined are as follows:

1. Area to be determined:

Tag size, Dipole size and T-matching size

2. Length to be determined:

T-matching line width, Distance between central lines of dipole and T-matching line

3. Impedance to be determined:

Impedance formed from the T-matching stripline and the dipole stripline, Dipole impedance, Chip impedance

4. Other:

Wave number - number of waves in a unit distance, K 
Aaron Don M. Africa et al., International Journal of Emerging Trends in Engineering Research, 8(9), September 2020, 5321 - 5326

\section{DATA AND RESULTS}

For the simulation MATLAB [13] was used as the tool. Figures 4 to 14 shows the output of the MATLAB simulation of the system.

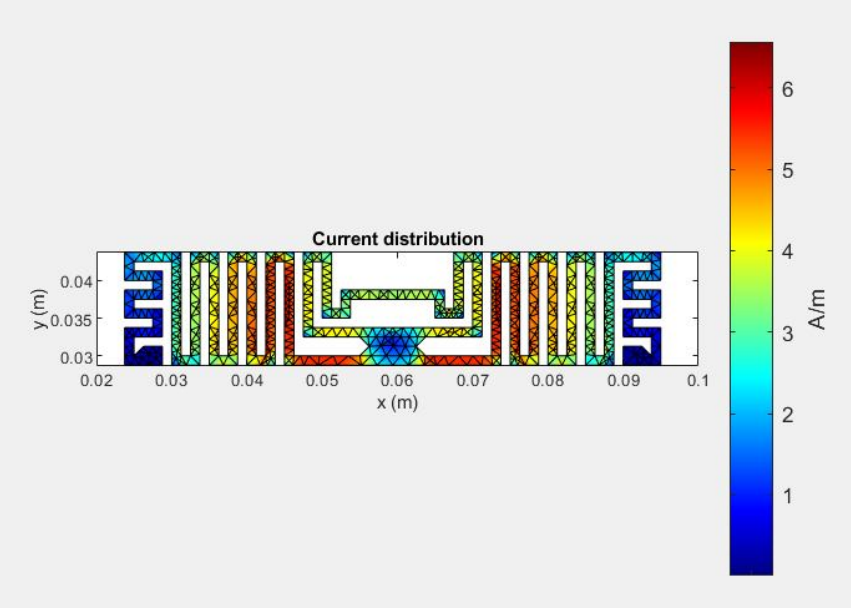

Figure 4: Stripline Current Distribution

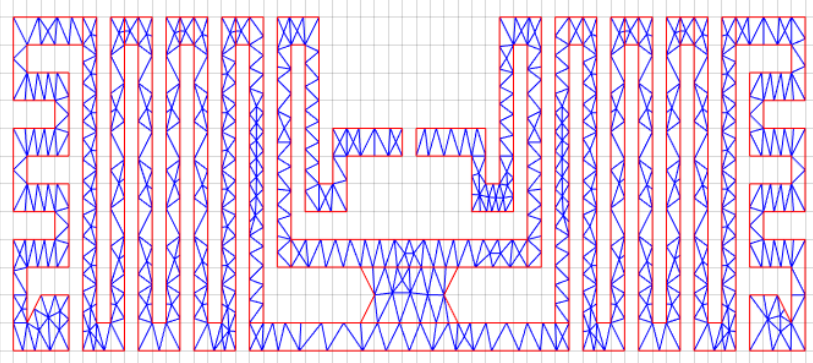

Figure 5: RFID Mesh Design

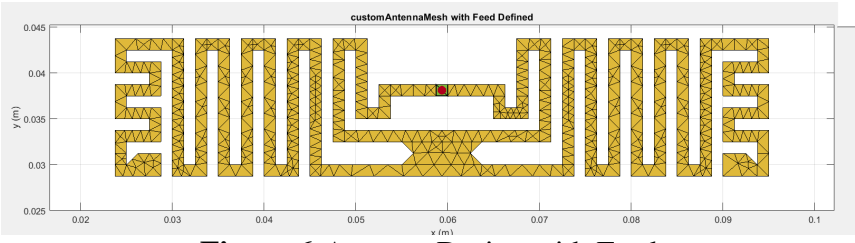

Figure 6: Antenna Design with Feed

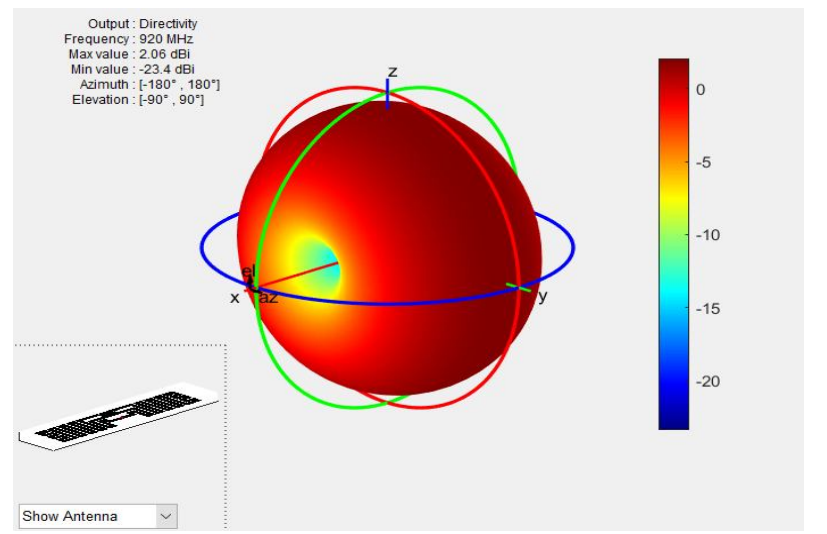

Figure 7:Radiation Pattern

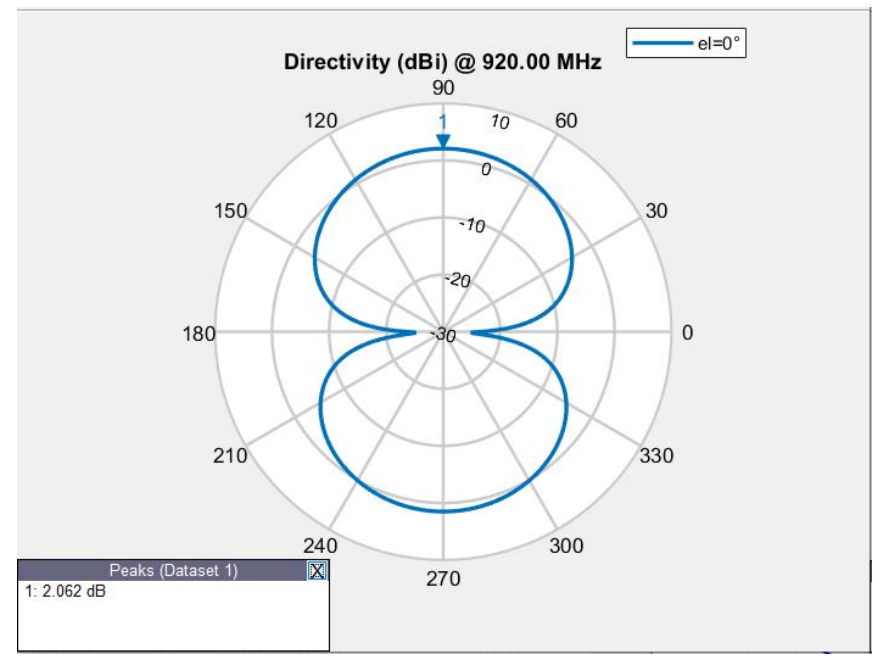

Figure 8: Radiation Pattern at Azimuth Angle $=0^{\circ}$

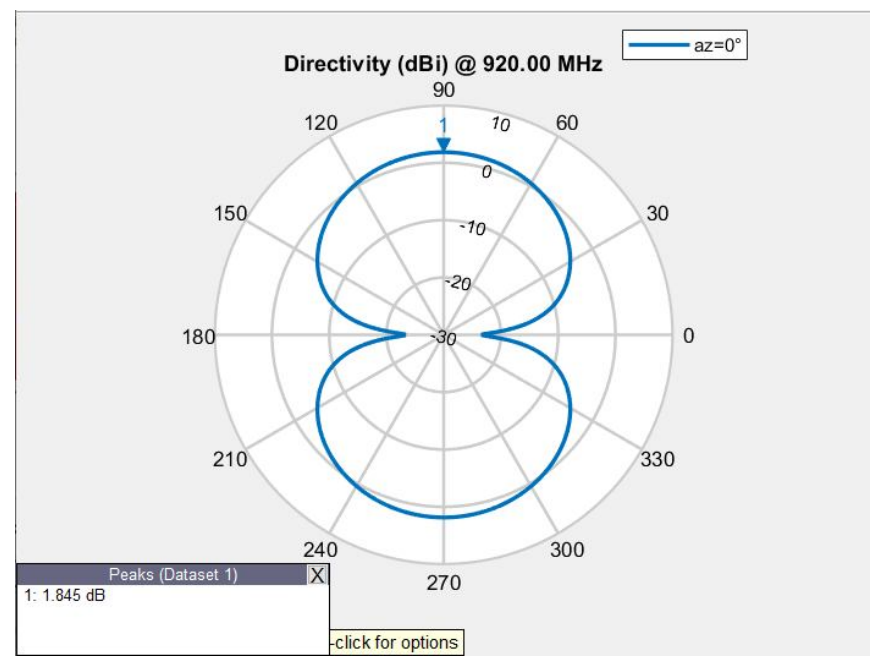

Figure 9: Radiation Pattern at Elevation Angle $=0^{\circ}$

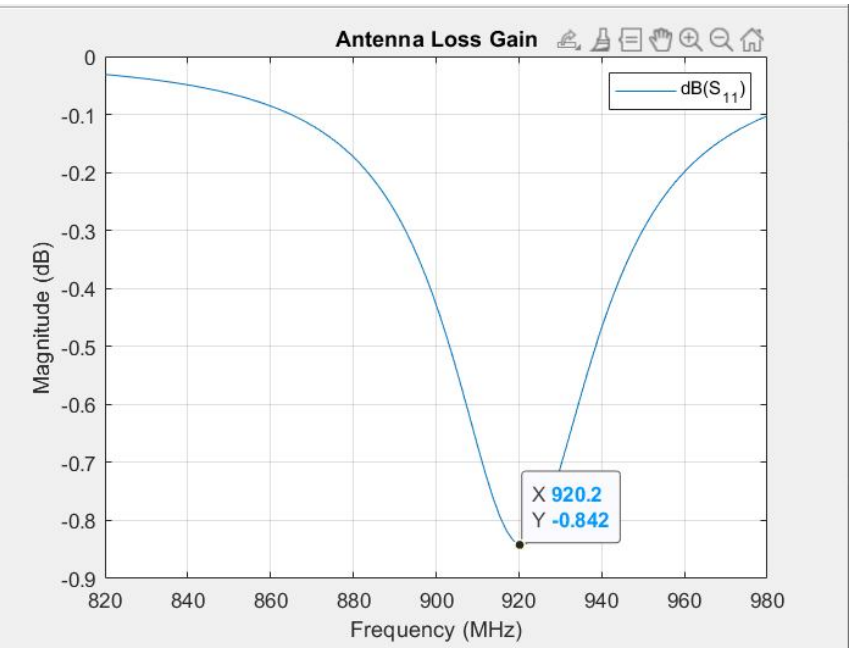

Figure 10: Antenna Loss Gain at $920 \mathrm{MHz}$ 


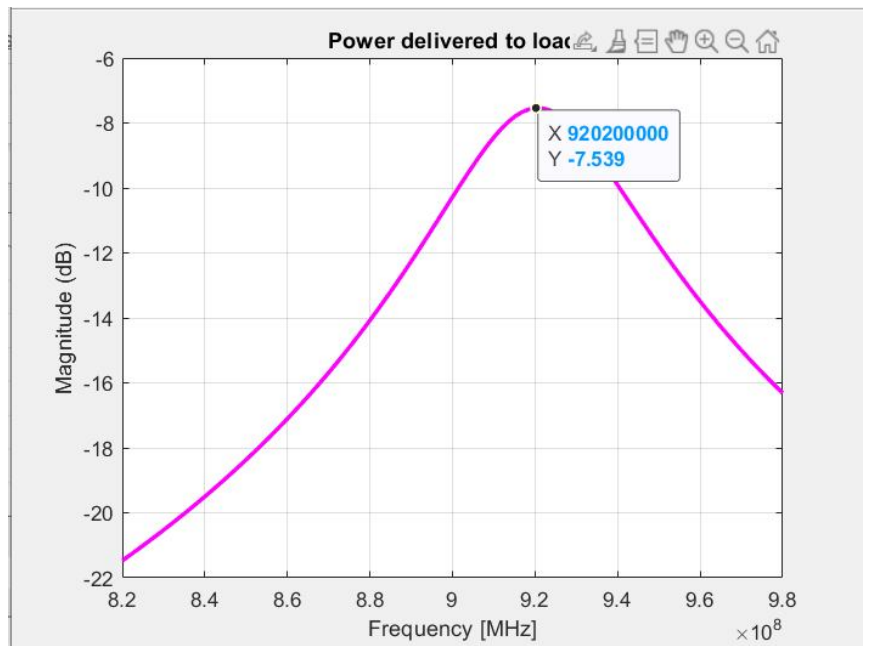

Figure 11:Power delivered to Load at $920 \mathrm{MHz}$

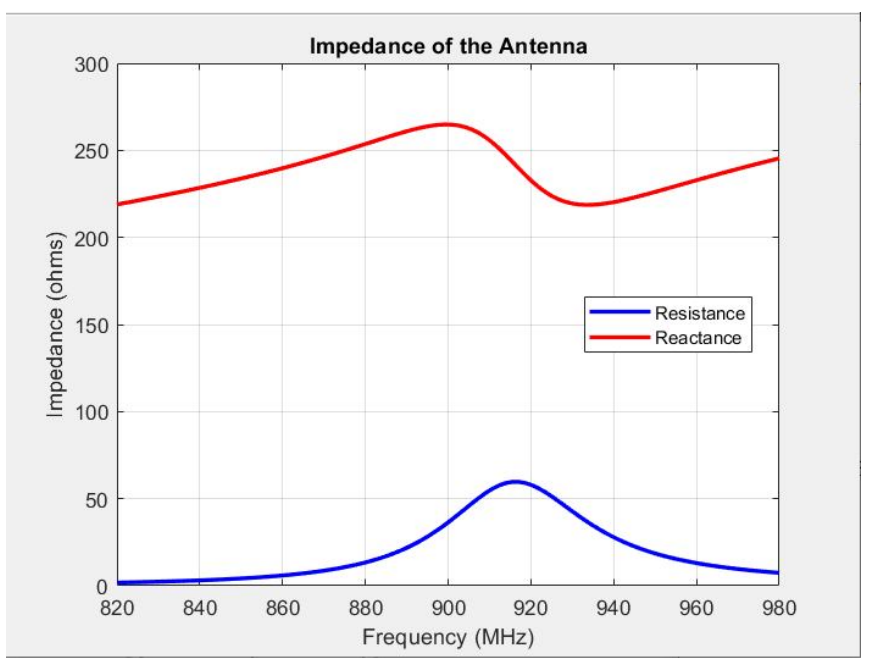

Figure 12: Impedance of the Antenna

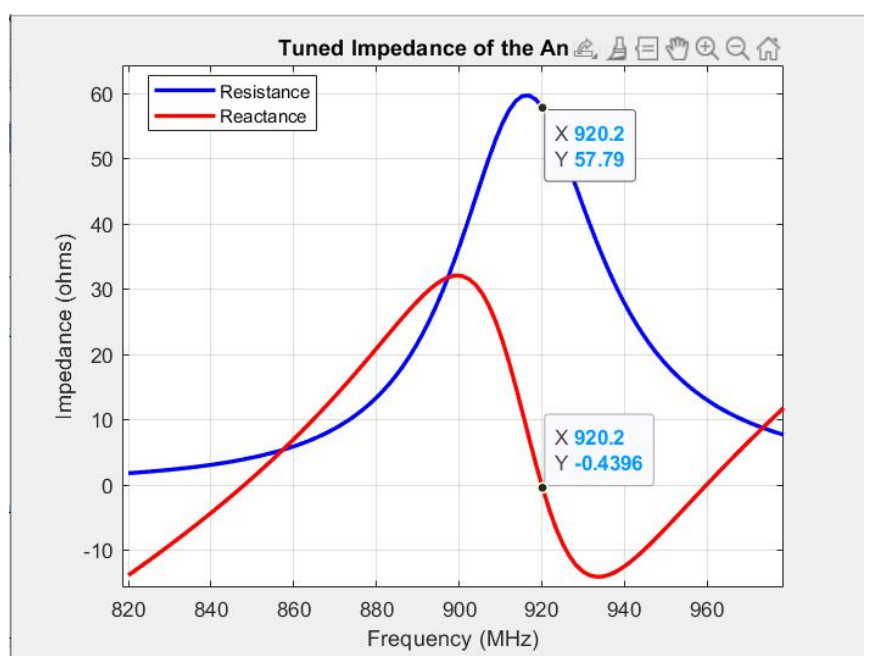

Figure 13: Matched Impedance after Tuning

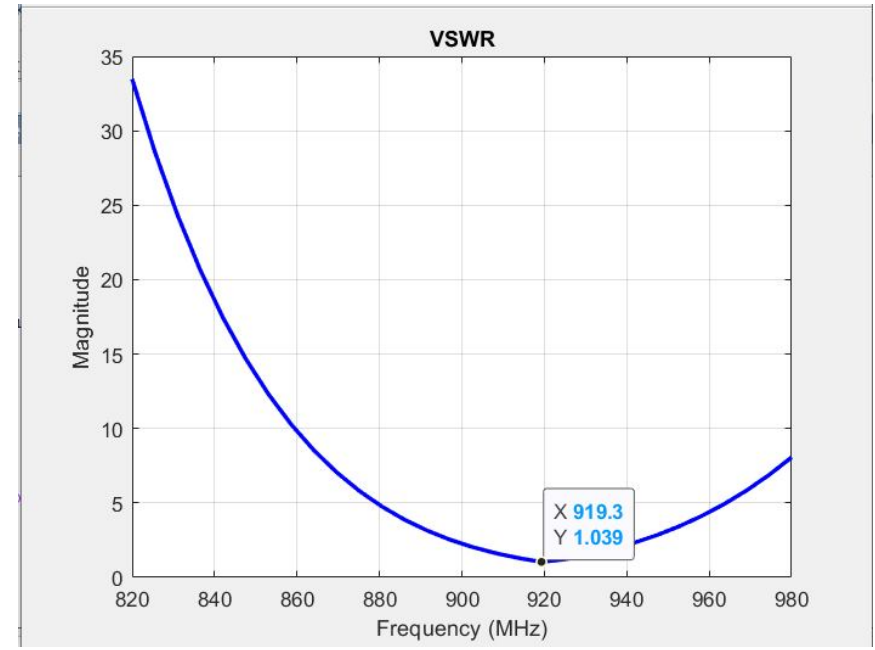

Figure 14: Voltage Standing Wave Ratio

\section{ANALYSIS OF DATA}

The performance of tag antennas directly affects the range and the quality of use of RFID systems. MATLAB can handle the parameters needed to be identified. One of the features of MATLAB is to navigate the strength of current distributed in the antenna. This allows them to understand the effectiveness of the variants of impedance on different sections of the antenna. With supported knowledge, PDE tool is usable in design a meshes needed for the tag. In conjunction of the Antenna Toolbox, the mesh can be converted into a custom antenna with a feed. This feed has preset microchips that have properties that are configurable $[14,15]$. This has been done in the experiment, changing the load impedance of the feed so that it can acquire the best power transfer system possible. The radiation pattern is also provided by MATLAB. This is essential in antenna design to identify the directivity of the antenna. RFID Antenna nowadays have wide-range due to their omni directivity. The radiation pattern feature of the programming platform also details the antenna gain and loss. These parameters are the defining characteristics of the antenna and are the main reason how antennas work. Impedance is also calculated and analyzed in the simulation. Based on the custom mesh antenna, the platform has visualized the antenna with proper properties akin to a RFID antenna. This results in obtaining other parameters in the frequency domain such as power delivered to the load and VSWR.

\section{CONCLUSION}

There are several considerations in designing and analyzing RFID Tag. As the core unit employed in several systems such as IoT and security network, its effectiveness need not to be undermined. Thanks to the advancement of technology, configuration of these necessities has been well-developed and cultured at the point where it is to understand and adduce the information to design and analyze given the platform. It is now easier to identify and to contribute to antenna design with efficiency and objectivity. 
For generating the various types of antenna designs simulations, MATLAB is a well-suited computer program, with optimal functions and command. With the potential to be applied in different scenarios, the creators of the tool installed an easy way to generate the different types of signals of these diagrams. This helps engineers and/or users of the tool to efficiently do their jobs.

In generating the different types of signals and functions of these diagrams, it is important to correct data in order to decrease the possibility of getting an error in the plots. Properly learning the language is required in order to make the least number of mistakes possible in creating a code that provides the required output.

\section{RECOMMENDATIONS}

There are prominent and newer studies that may contribute to generating new algorithms in matching antenna impedance. Dividing the RFID antenna dimensions into several types of antennas is surely an expressive way of delving into antenna designing. The idea is to pinpoint the inductive antenna stripline dimension and the meander antenna stripline dimensions to compute its impedance.

\section{REFERENCES}

[1] S. Anandhi, R. Anitha, and V. Sureshkumar, "An Authentication Protocol to Track an Object with Multiple RFID Tags using Cloud Computing Environment," Wireless Personal Communications, vol. 113, no. 4, pp. 2339-2361, 2020.

[2] N. Sghaier and L. Latrach, "Iterative Technique for Analysis and Design of Circular Leaky-Wave Antenna for the 2.45-GHz Radio-Frequency Identification Applications," International Journal of RF and Microwave Computer-Aided Engineering, vol. 30, no. 7, 2020.

[3] J. Teo,A. Loganathan, P. Goh, and N. S. Ahmad, "Autonomous Mobile Robot Navigation Via RFID Signal Strength Sensing," International Journal of Mechanical Engineering and Robotics Research, vol. 9, no. 8, pp. 1140-1144, 2020.

[4] M. El-Absi,F. Zheng, A. Abuelhaija, A. -H Abbas, K. Solbach, and T. Kaiser, "Indoor Large-Scale Mimo-Based Rssi Localization with Low-Complexity Rfid Infrastructure," Sensors (Switzerland), vol. 20, no. 14, pp. 1-30, 2020.

[5] C. Luo,I. Gil, and R. Fernández-García, "Wearable Textile UHF-RFID Sensors: A Systematic Review," Materials, vol. 13, no. 15, 2020.

[6] F. Babaeianand N. C. Karmakar, "Development of Cross-Polar Orientation-Insensitive Chipless RFID Tags," IEEE Transactions on Antennas and Propagation, vol. 68, no. 7, pp. 5159-5170, 2020.

[7] R. Polvara,M. Fernandez-Carmona, G. Neumann, G. Neumann, G. Neumann, and M. Hanheide, "Next-Best-Sense: A Multi-Criteria Robotic Exploration
Strategy for RFID Tags Discovery," IEEE Robotics and Automation Letters, vol. 5, no. 3, pp. 4477-4484, 2020.

[8] E. Hatem, S. Abou-Chakra, E. Colin, J. -M Laheurte, and B. El-Hassan, "Performance, Accuracy and Generalization Capability of Rfid Tags' Constellation for Indoor Localization," Sensors (Switzerland), vol. 20, no. 15 pp. 1-15, 2020.

[9] B. Pawłowicz, B. Trybus, M. Salach, and P. Jankowski-Mihułowicz, "Dynamic RFID Identification in Urban Traffic Management Systems," Sensors (Switzerland), vol. 20, no. 15, pp. 1-26, 2020.

[10]E. Zahran,A. Arafa, H. Saleh, and M. I. Dessouky, “A Self Learned Invasive Weed-Mixed Biogeography Based Optimization Algorithm for RFID Network Planning," Wireless Networks, vol. 26, no. 6, pp. 4109-4127, 2020.

[11] T. Das,B. Dwivedy, D. Behera, S. K. Behera, and N. C. Karmakar, "Design and Modelling of a Compact Circularly Polarized Antenna for RFID Applications," AEU - International Journal of Electronics and Communications, vol. 123, 2020.

[12]Z. Hailemariam, Y. -C Lai, R. Jayadi, Y. -H Chen, and S. -C Huang, "A Knowledge-Based Query Tree with Shortcutting and Couple-Resolution for RFID Tag Identification," Computer Communications, vol. 160, pp. 779-789, 2020.

[13] Matlab. https://www.mathworks.com/products/matlab.html. 2020.

[14] A. Africa, R. Manuel, J. Ligayo, J. Tiberio, and R. Munchua, "Design of a low-power smart antenna system algorithm," International Journal of Emerging Trends in Engineering Research, vol. 8, no. 8, pp. 4443-4448, 2020.

[15] M. Said, M. Misran, Z. Zakaria, M. Zin, and L. Muhammad, "Design of a circularly polarized antenna at $2.45 \mathrm{ghz}$ with harmonic suppression for material characterization," International Journal of Emerging Trends in Engineering Research, vol. 8, no. 8, pp. 4420-4425, 2020. 